\title{
3rd Early Career Issue
}

\author{
J. Vermant $\cdot$ H. H. Winter
}

Published online: 23 November 2014

(C) Springer-Verlag Berlin Heidelberg 2014

Rheologica Acta tries to be at the forefront of the field of rheology. Last year, we started providing researchers in the early stages of their career the possibility to contribute an invited paper to a special issue of Rheologica Acta. These papers are original contributions, which underwent the regular reviewing process, but the authors were given a bit more freedom to speculate about the impact of their work. Following the success of last year's issues, we selected a few authors and invited them to do same. In this issue, you will find the fruits of their work, giving us a clear view of the topics, which are shaping up our discipline for the years to come.

Lerouge and coworkers present a cautionary tale on the use of large amplitude oscillatory shear flows (LAOS), which represent complex kinematic conditions and even for Newtonian fluids secondary flows caused by inertia can complicate matters. When this is combined with a system prone to elastic instabilities, complex flow patterns will emerge (Fardin et al. 2014). Bozorgi and Underhill (2014) apply LAOS to swimming bacteria, which are a class of active suspensions with some different nonlinear responses compared to the traditional passive suspensions. These authors also demonstrate how rheological fingerprinting of such active suspension may be obtained from the nonlinear material functions. Ardekani and coworkers tackle a different problem in swimming bacterial suspensions (Li et al. 2014). Whereas most work so far has focused on bacteria swimming in Newtonian media, most

J. Vermant $(\bowtie)$

Department of Materials, ETH Zürich, 8093 Zürich, Switzerland

e-mail: Jan.vermant@mat.ethz.ch

H. H. Winter

Department of Chemical Engineering, University of Massachusetts, Amherst, MA 01003, USA

e-mail: winter@ecs.umass.edu bacteria live in environments, which contain polymers and DNA fragments which make the local environment viscoelastic. As a consequence, the hydrodynamic interactions will change. This is addressed here numerically by using a Giesekus model. The effect of both shear thinning and elastic effects is investigated numerically. Cha and coworkers (2014) focus on viscoelastic effects in the area of microfluidics, where the normal stress differences generated in shear flow are exploited to focus particles in the center of a channel, even in the absence of inertia.

Snijkers and Vlassopoulos (2014) investigate how an often used empirical rule in the rheological literature, the well-known Cox-Merz (CM) rule, holds up when applied to modern, molecularly engineered polymers. Even for more complex architectures, the rule is found to be observed surprisingly well. Failures to obey the $\mathrm{CM}$ rule are observed for branched polymers, and the relevance of this observation is discussed. Finally, Koos et al. (2014) report on exiting new types of suspensions, where attractive interactions between the particles are induced by thin capillary bridges between the particles created by a small amount of immiscible fluids. Such suspensions show all the hallmark features of aging systems and seem to be well suited to study these phenomena in depth.

We thank the authors for their fine contributions to the journal and hope that this collection once again reflects important trends in rheology. We wish you enjoyable reading.

\section{References}

Bozorgi Y, Underhill PT (2014) Large-amplitude oscillatory shear rheology of dilute active suspensions. Rheol Acta doi:10.1007/s00397014-0806-y 
Cha S, Kang K, You JB, Im SG, Kim Y, Kim JM (2014) Hoop stress-assisted three-dimensional particle focusing under viscoelastic flow. Rheol Acta doi:10.1007/s00397-0140808-9

Fardin MA, Perge C, Casanellas L, Hollis T, Taberlet N, Ortín J, Lerouge S, Manneville S (2014) Flow instabilities in large amplitude oscillatory shear: a cautionary tale. Rheol Acta doi:10.1007/s00397-0140818-7
Koos E, Kannowade W, Willenbacher N (2014) Restructuring and aging in a capillary suspension. Rheol Acta doi:10.1007/s00397-014-0805-Z

Li G-J, Karimi A, Ardekani AM (2014) Effect of solid boundaries on swimming dynamics of microorganisms in a viscoelastic fluid. Rheol Acta doi:10.1007/s00397-014-0796-9

Snijkers F, Vlassopoulos D (2014) Appraisal of the Cox-Merz rule for well-characterized entangled linear and branched polymers. Rheol Acta doi:10.1007/s00397-014-0799-6 